\title{
Investigation of the Electro-Mechanical Behavior of Hybrid Polyaniline/Graphene Nanocomposites Fabricated by Dynamic Interfacial Inverse Emulsion Polymerization
}

\author{
Rami Regueira ${ }^{1,2}$, Ran Yosef Suckeveriene ${ }^{2,3}$, Irena Brook ${ }^{1,2}$, Guy Mechrez ${ }^{2}$, \\ Roza Tchoudakov ${ }^{2}$, Moshe Narkis ${ }^{2 *}$ \\ ${ }^{1}$ Interdepartmental Program in Polymer Engineering, Technion-IIT, Haifa, Israel \\ ${ }^{2}$ Department of Chemical Engineering, Technion-IIT, Haifa, Israel \\ ${ }^{3}$ Department of Water Industries Engineering, Kinneret College in the Jordan Valley, Zemach, Israel \\ Email:
}

Received 30 December 2014; accepted 18 January 2015; published 26 January 2015

Copyright (C) 2015 by authors and Scientific Research Publishing Inc.

This work is licensed under the Creative Commons Attribution International License (CC BY). http://creativecommons.org/licenses/by/4.0/

(c) $\underset{\mathrm{EY}}{\mathbf{0}}$ Open Access

\section{Abstract}

This paper describes a study on electrical resistivity under loading of polyaniline (PANI)/graphene nanocomposite powders and compacts. The composites were prepared by an in-situ interfacial dynamic inverse emulsion polymerization technique under sonication of aniline in the presence of graphene sheets in chloroform. During polymerization the graphene nanoplatelets are coated with PANI and are well dispersed both in the polymeric suspension and then in the dried polymer matrix as evidenced by cryogenic transmission electron microscopy (Cryo-TEM) and high resolution scanning microscopy (HRSEM). The presence of graphene nanoplatelets lowers the electrical resistivity of the polyaniline by two orders of magnitude for both the powder and the compact composites as demonstrated by their electrical resistance measurements conducted under loading. The lowest measured electrical resistivity values were $5 \Omega \cdot \mathrm{cm}$ for $33 \% \mathrm{wt}$. graphene powder and $8 \Omega \cdot \mathrm{cm}$ for $41 \%$ wt. graphene compacted composites. Cyclic electrical measurements under loading showed a distinct reproducible dependence of the bulk resistivity vs. applied pressure. This repetition is a key component for electro-mechanical sensors. To the authors' best knowledge, this is the first report on polymerization of aniline in presence of graphene by the in-situ interfacial dynamic inverse emulsion polymerization technique and also the first report on cyclic electrical measurements under pressure of PANI/graphene nanocomposites.

\section{Keywords}

Nanocomposites, Polyaniline, Graphene, Electro-Mechanical Sensors, Inverse Emulsion

\footnotetext{
${ }^{*}$ Corresponding author.
} 


\section{Polymerization}

\section{Introduction}

Fillers, or additives, are commonly used to modify polymer properties and often upgrade their performance [1] [2]. Incorporation of nano-sized fillers, in polymers is commonly referred to as nanocomposites. Nanocomposites have attracted great interest due to their wide possible range of applications and commercial opportunities [3]-[7].

One of the most promising nanofillers is graphene [8]-[10]. Graphene is a flat monolayer of carbon atoms tightly packed into a two-dimensional (2D) honeycomb lattice [8]. Graphene offers excellent properties such as: electrical conductivity, good mechanical properties and flexibility [1] [11]. Thus, graphene is widely used as an interesting alternative for carbon-based nanofillers in developing new nanocomposites [12].

Polyaniline (PANI) is one of the most studied intrinsically conductive polymers (ICP) [13] [14]. It possesses unique properties such as high electric conductivity, thermal transport and environmental stability, but yet it is simple to produce [15]. PANI can be produced in an organic, or an aqueous phase, using stirring [16] or sonication [17], static interfacial [18], emulsion [19] and inverse emulsion [20] polymerization methods.

The combination of the unique physical properties of graphene incorporated into polymer matrices and PANI has been a focus of research in a wide variety of applications, including: a novel electrode material for high performance super-capacitors [21], electrochemical sensing [22] and bio-sensing [23].

Graphene sheets have a high tendency to agglomerate due to strong van der Waals and $\pi$ - $\pi$ interactions. Many approaches have been discussed in the literature to reduce agglomeration such as covalent [24] and non-covalent [25] chemical functionalization, attachment of surfactants [26] and grafting polymers onto the graphene nanoplatelets [27]. A successful approach for agglomerates breakdown is in-situ polymerization of monomers in the presence of nanoparticles [7]. Emulsion polymerization is a common technique for such reactions which usually comprise a micelle-forming surfactant, a water-soluble initiator in combination with a water insoluble monomer. In this technique the monomer is dispersed, with the aid of a surfactant, within organic micelles which are well dispersed in the continuous aqueous phase. When adding the initiator, reaction occurs and the product is usually a stable latex, a submicron dispersion of polymer particles in water [28] [29]. Inverse emulsion polymerization is less common, but well known polymerization technique. This technique comprises a continuous organic phase with dispersed water droplets. Inverse emulsion polymerization can be used for various monomers, including aniline [13] [30].

The mechanical properties of confined compressed powders have drawn much attention for the production of pharmaceutical products, mainly in the development of formulations [31], metallurgy science [32] and sensors [33] [34]. The ability to estimate the volume reduction or density change of powders as a result of applied pressure, or shock waves, is of significant industrial importance. Kawakita et al. [35] have suggested an empirical model to predict the volume reduction of the powdered particles under compression as a function of the applied pressure.

Sensors are used to convert a measured physical dimension into a change of an electrical property that can be transmitted electrically meaning they create a coupling between mechanical and electrical energy [36]. Electromechanical sensors transmit the applied mechanical force into an electrical signal. Such sensors are of high importance in non-destructive testing.

Carbon nanotubes (CNT) and graphene are promising candidates for electromechanical sensors. Both materials exhibit a piezoresistivity behavior in tensile and compression loading, when present in a polymer matrix [37] or fabricated as free standing hybrid sheets [38]. In addition, cyclic tensile loading experiments for CNT/ $\mathrm{Al}_{2} \mathrm{O}_{3} /$ epoxy [39] and CNT/vinyl ester [40] composites have also demonstrated self-sensing capabilities.

This work describes a novel technique for fabrication of PANI/graphene nanocomposites by an inverse emulsion polymerization technique under sonication, of aniline in a presence of graphene nanoplatelets. Polyaniline coats the graphene surface, resulting in a good dispersion. The addition of graphene sheets lowers the composite electrical resistivity. Cyclic loading experiments of the compacts have shown consistent, direct and reproducible connection to resistivity, demonstrating that the composite can be used as a sensing material. This paper describes a unique novel approach for the production of an active element for electro-mechanical sensors based on 
hybrid nanocomposites PANI/graphene prepared by using the inverse emulsion polymerization technique.

\section{Experimental}

\subsection{Materials}

Aniline monomer (Aldrich, USA) was used after purification. Dodecyl benzene sulfonic acid (DBSA) (Zohar, Israel) was used as received without further purification. Chloroform (Bio Lab LTD., Israel) was used as a solvent. A selected grade of graphene AO-3 with average flake thickness of $12 \mathrm{~nm}$ and average particle size of 1.5 $10 \mu \mathrm{m}$ (Graphene Nanopowder, USA) was studied. Ammonium peroxydisulfate (APS) was used as received (Riedel de-Haën, Sigma-Aldrich, Germany). Ethyl-alcohol (Gadot, Israel) was used for a precipitation procedure of the dispersions and also for washing residues. Vibracell VCX 750 (Sonics \& Materials Inc., USA) ultrasonic liquid processor was used to disperse the graphene sheets prior to polymerization.

\subsection{Preparation of Nanocomposites}

The inverse emulsion polymerization procedure of aniline in organic solvents was performed as previously reported [7] [20] [29]. A certain amount of the dopant (DBSA) is dissolved in $200 \mathrm{ml}$ chloroform using magnetic stirring. Distilled aniline is added and mixed until a clear solution is formed. The graphene nanoparticles are added to the solvent and sonicated for 5 min to disperse the graphene sheets. Graphene concentration is typically in the range of $0.05 \%-0.7 \%$ wt. of the dispersion. APS dissolved in $10 \mathrm{ml}$ distilled water, is added to the solvent/aniline solution followed by sonication at $4^{\circ} \mathrm{C}$ for $15 \mathrm{~min}$. The molar ratio of aniline:dopant:APS is 1:1:0.25.

The resulting PANI/graphene dispersions were used for the formation of nanocomposites using a precipitation-filtration technique. A typical precipitation-filtration procedure is performed as follows: The dispersion is precipitated in ethyl-alcohol followed by gravitational filtration. The formed nanocomposites are washed with distilled water, the wet composites are dried in an oven at $80^{\circ} \mathrm{C}$ for $3 \mathrm{hr}$ followed by additional drying under vacuum at $80^{\circ} \mathrm{C}$ overnight.

Two forms of samples were prepared from the resulting powdered composites. A certain amount of the resulting composites was ground to achieve a fine powder for further characterization as powder sample. Another amount of the powder was compressed under pressure of $\sim 6$ MPa during 10 min to achieve powder compacts which were also studied.

Reference samples, without graphene, were prepared using the same procedure.

\subsection{Characterization}

The structure of the PANI/graphene nanocomposites and their dispersion quality were investigated by cryogenic transmission electron microscopy (Cryo-TEM) as described by Talmon et al. [41]. The TEM micrographs were obtained for ultra-fast cooled vitrified Cryo-TEM specimens prepared under controlled conditions at $20^{\circ} \mathrm{C}$ and $100 \%$ relative humidity. Specimens were examined in a Philips CM120 Cryo-TEM operating at $120 \mathrm{kV}$, using an Oxford CT3500 cooling-holder system at about $-180^{\circ} \mathrm{C}$. Low electron-dose imaging was performed with a Gatan Multiscan 791 CCD camera, using the Gatan Digital Micrograph 3.1 software package.

The morphology of the PANI/graphene nanocomposites was studied using a high resolution scanning electron microscopy (HRSEM, Zeiss Ultra Plus), equipped with a high-resolution field emission gun (FEG), operated at a $4 \mathrm{kV}$ accelerating voltage in a $3-5 \mathrm{~mm}$ working distance, and an in-lens detector of secondary electrons. The compacts were freeze-fractured in liquid nitrogen and carbon sputtered prior to observation. The composites obtained were further characterized by dripping a small amount of PANI/graphene dispersion on $\mathrm{SiO}_{2}$ wafer. After solvent evaporation, the sample was dried under vacuum for $24 \mathrm{hr}$ at $60^{\circ} \mathrm{C}$.

FTIR spectrometer was used to characterize chain interactions. Absorbance infrared Fourier-transform spectra were recorded using a thermo 6700 FT-IR equipped with a Smart Itr diamond ATR device. Each spectrum was recorded at a resolution level of $4 \mathrm{~cm}^{-1}$.

Samples were analyzed using a TA 2050 Thermal Gravimetric Analyzer (TGA). Samples were heated under air, at a heating rate of $20^{\circ} \mathrm{C} / \mathrm{min}$., monitoring their weight loss as a function of temperature.

Surface area of the PANI/graphene nanocomposites was studied by the BET method.

Mechanical properties were evaluated using an Instron (5568) universal testing machine at a crosshead speed 


\section{of $3 \mathrm{~mm} \cdot \mathrm{min}^{-1}$.}

Compression measurements were carried out using a round chamber, $5 \mathrm{~cm}$ diameter, under maximum a load of 1.6 MPa. Electrical measurements were recorded simultaneously using a Keithley (6510) instrument.

Bulk resistivity measurements of the compressed compact composites were done using a Keithley 175A instrument.

\section{Results and Discussion}

Figure 1 depicts cryogenic transmission electron microscopy (Cryo-TEM) images of: Figure 1(a) neat PANI and Figure 1(b) PANI with 0.5\% wt. graphene dispersions in chloroform. Neat PANI (Figure 1(a)) exhibits typical spherical particles with mean diameter of $\sim 100 \mathrm{~nm}$. A good dispersion of the graphene sheets in the PANI dispersion is shown in Figure 1(b). Thus, it is suggested that since PANI is partially miscible in chloroform, it coats the graphene sheets and well disperses them. A similar behavior was reported by Suckeveriene et al. [7] and KIM et al. [42] with carbon nanotubes (CNT).

Figure 2 depicts HRSEM micrographs of dry: Figure 2(a) neat PANI, Figure 2(b) PANI with 20\% wt. graphene dispersions deposited on $\mathrm{SiO}_{2}$ wafers. Neat PANI, shown in Figure 2(a), depicts spherical particles (similar to Figure 3(a)) which are typical of neat PANI. Figure 2(b) demonstrates a well polyaniline coating of the graphene nanoplatelets as marked by the arrow, PANI completely covers the entire graphene sheets. Similar PANI coating of carbon nanotubes was reported by Suckeveriene et al. [7]. The polyaniline coating is attributed to the fine graphene dispersion.

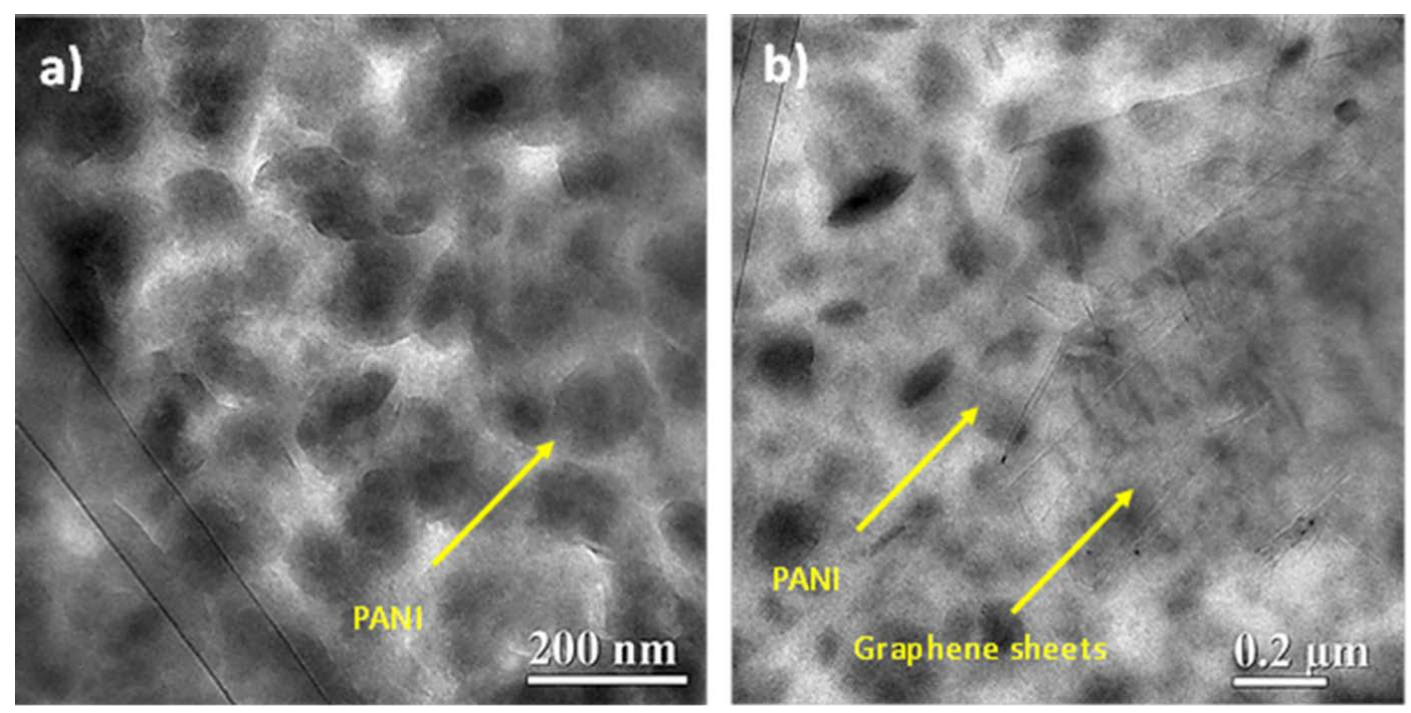

Figure 1. Cryo-TEM images of dispersions: (a) Neat PANI; (b) PANI with 0.5\% wt. graphene.
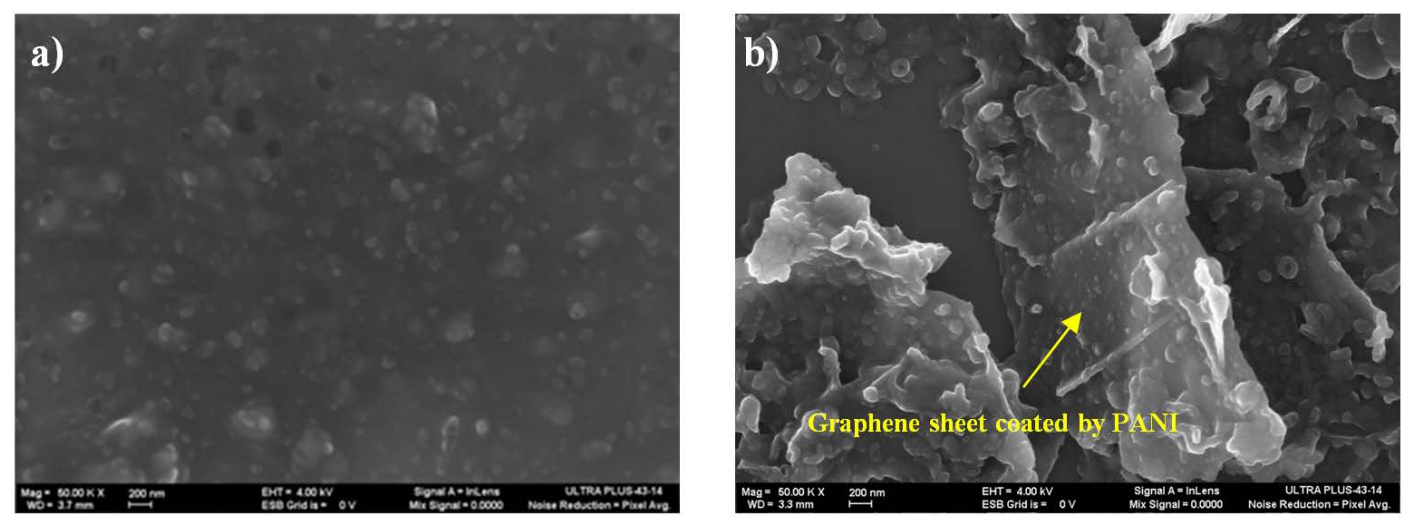

Figure 2. HRSEM images of dispersions deposited on $\mathrm{SiO}_{2}$ wafers: (a) Neat PANI; (b) PANI with $20 \%$. 

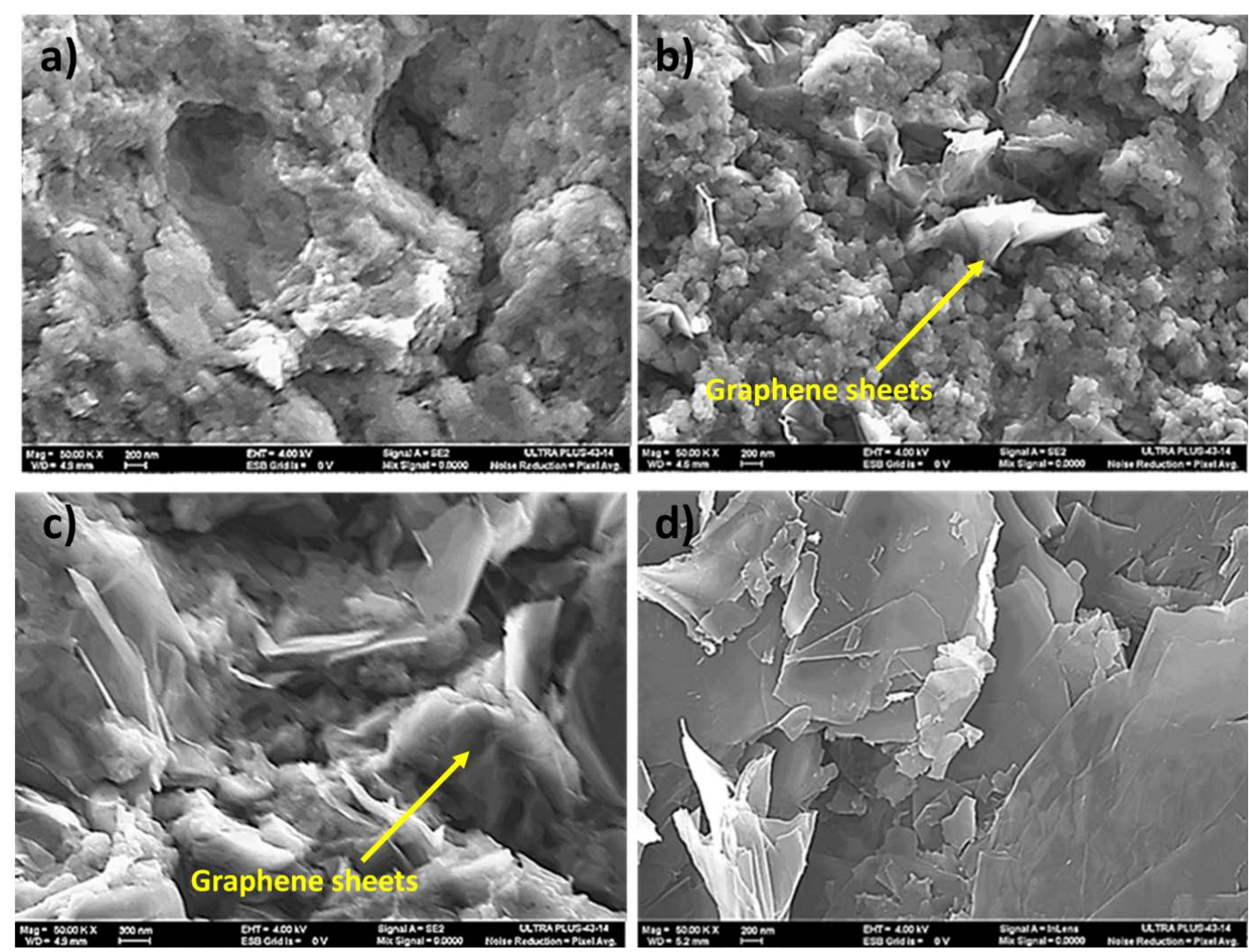

Figure 3. HRSEM cross-section images of composite compacts: (a) Neat PANI; (b) PANI with 5\% wt. graphene; (c) PANI with 23\% wt. graphene; (d) Neat graphene.

Figure 3 depicts freeze fractured compacts HRSEM micrographs of: Figure 3(a) neat PANI, Figure 3(b) PANI with 5\% wt. graphene, Figure 3(c) PANI with 23\% wt. graphene and Figure 3(d) neat graphene. The images are perpendicular to the pellet's plane. Neat PANI, shown in Figure 3(a), depicts spherical particles typical of aniline polymerization with DBSA [43]. Figure 3(b), Figure 3(c) demonstrate well dispersed graphene sheets within the PANI matrix at two concentrations as marked by the arrows, and are well imbedded within the PANI matrix. Thus, the graphene is well dispersed in both the solvent and in the dry composites in agreement with Suckeverine [20]. Figure 3(d) depicts the graphene sheets mostly arranged in parallel planes orientation and stacked together.

Table 1 shows the surface area, measured using the BET technique, dependence on graphene content. Graphene surface area is $113.8 \mathrm{~m}^{2} \cdot \mathrm{g}^{-1}$ while neat PANI exhibits a lower value of $21.8 \mathrm{~m}^{2} \cdot \mathrm{g}^{-1}$. Since the surface area of samples with higher graphene contents (23\% and $41 \%$ ) is similar to the neat PANI, it further supports the conjecture that PANI efficiently covers and coats the graphene nanoplatelets' surface (as previously seen in Figure 2 \& Figure 3).

Figure 4 depicts FTIR spectra of neat graphene, neat PANI and PANI with 5\% wt. graphene. Graphene has a characteristic FTIR peak, as reported elsewhere [44] at $\sim 1509 \mathrm{~cm}^{-1}$ which is attributed to the skeletal vibration of graphene nano sheets. The PANI spectra presents characteristic FTIR peaks at $\sim 1530 \mathrm{~cm}^{-1}$ and $\sim 1390 \mathrm{~cm}^{-1}$, which correspond to $\mathrm{C}=\mathrm{N}$ stretching vibrations of the quinonoid ring and $\mathrm{C}-\mathrm{N}$ bond in the quinonoid-benzenoid, respectively. PANI/graphene exhibits similar peaks as the neat PANI and graphene, thus confirming that the inverse emulsion polymerization in the presence of graphene has resulted in PANI/graphene nanocomposites.

Figure 5 depicts TGA thermogram in air of neat PANI, PANI with $10 \%$ wt. graphene and PANI with 20\% wt. graphene. The TGA curves show, as expected, that as graphene content increases the thermal stability of the composite is higher since graphene is significantly more stable than PANI. Furthermore, the addition of graphene also produces a char barrier effect that prevents oxygen from reaching the material and thus lowering the burning rate and increasing thermal stability [45] [46]. 
Table 1. PANI/graphene composite powders BET surface area.

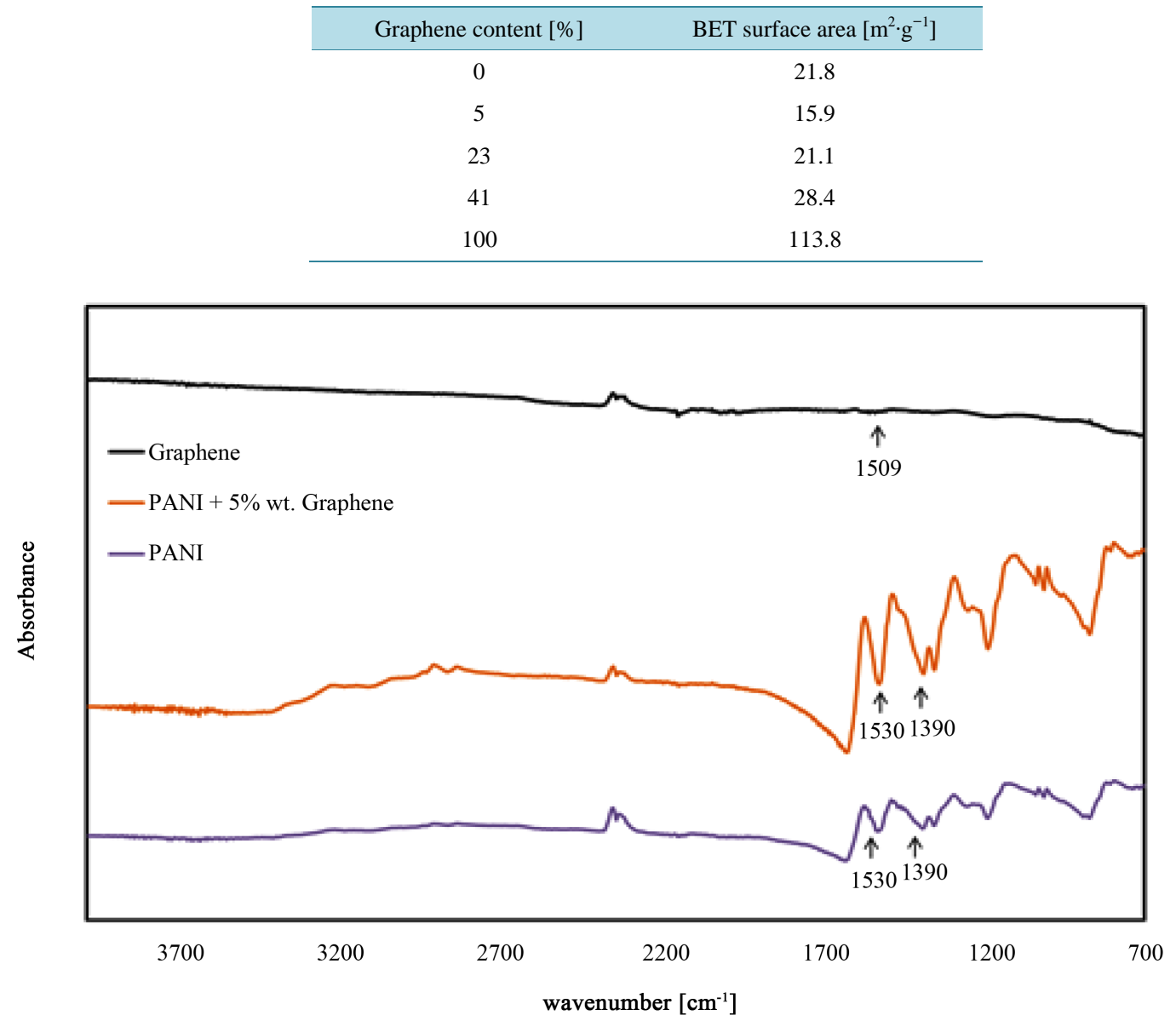

Figure 4. FTIR-ATR spectra of graphene, PANI and PANI with 5\% wt. graphene.

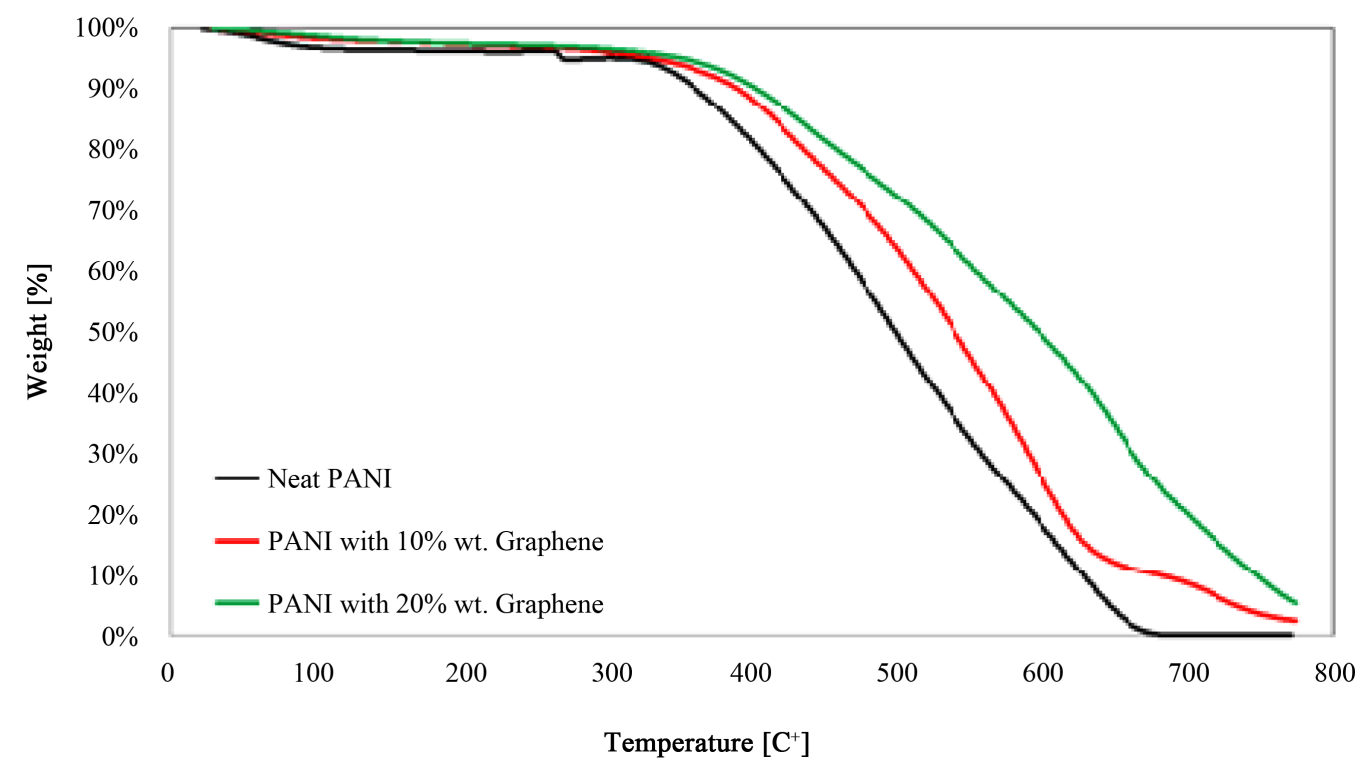

Figure 5. Thermal gravimetric analysis of PANI, PANI with $10 \%$ wt. graphene and PANI with $20 \%$ wt. graphene. 
Figure 6(a) depicts the volume resistivity of powders under compression up to a value of $\sim 1600 \mathrm{kPa}$. The volume resistivity significantly decreases down by two orders of magnitude from $\sim 79 \Omega \cdot \mathrm{cm}$ for neat PANI to $\sim 0.8 \Omega \cdot \mathrm{cm}$ for PANI with $33 \%$ wt. graphene in PANI at the end of the compression process. Furthermore it can be seen that the resistivity consistently decreases with the addition of graphene sheets. The higher graphene content resulted in lower resistivity values i.e., the graphene sheets significantly contribute to the increasing charge mobility of the composite by creating pathways for electric current conduction. In addition, with pressure increase the resistivity decreases, mainly due to better particles' contact [47] until a pseudo-plateau is obtained. The behavior observed for all samples, indicates that the powders are densified to highly compacted arrangements.

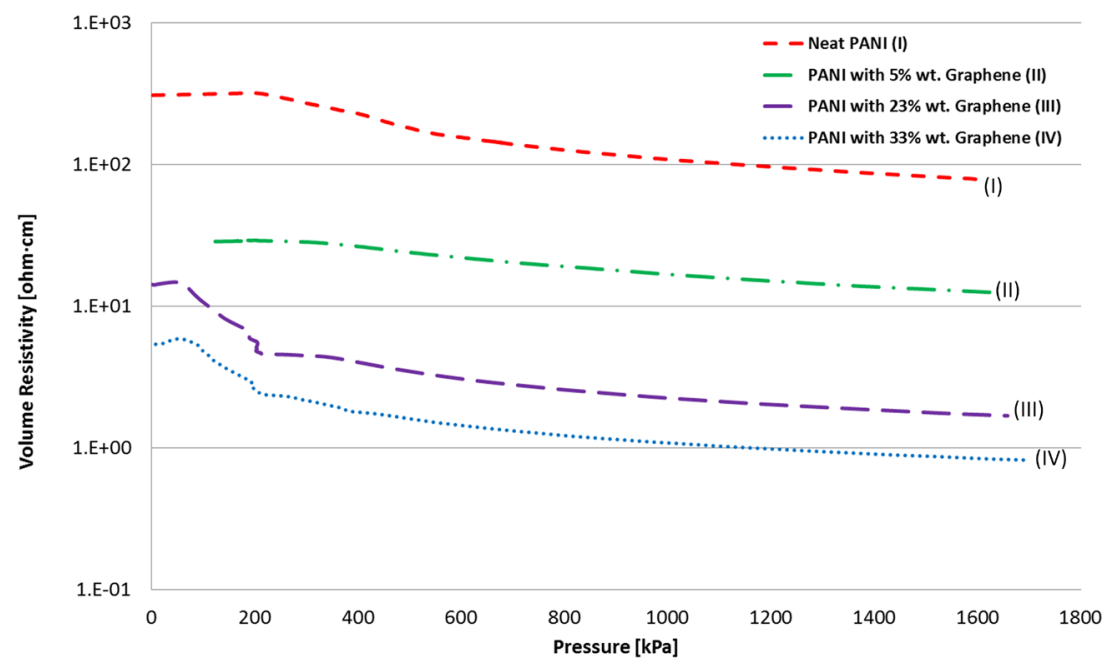

(a)

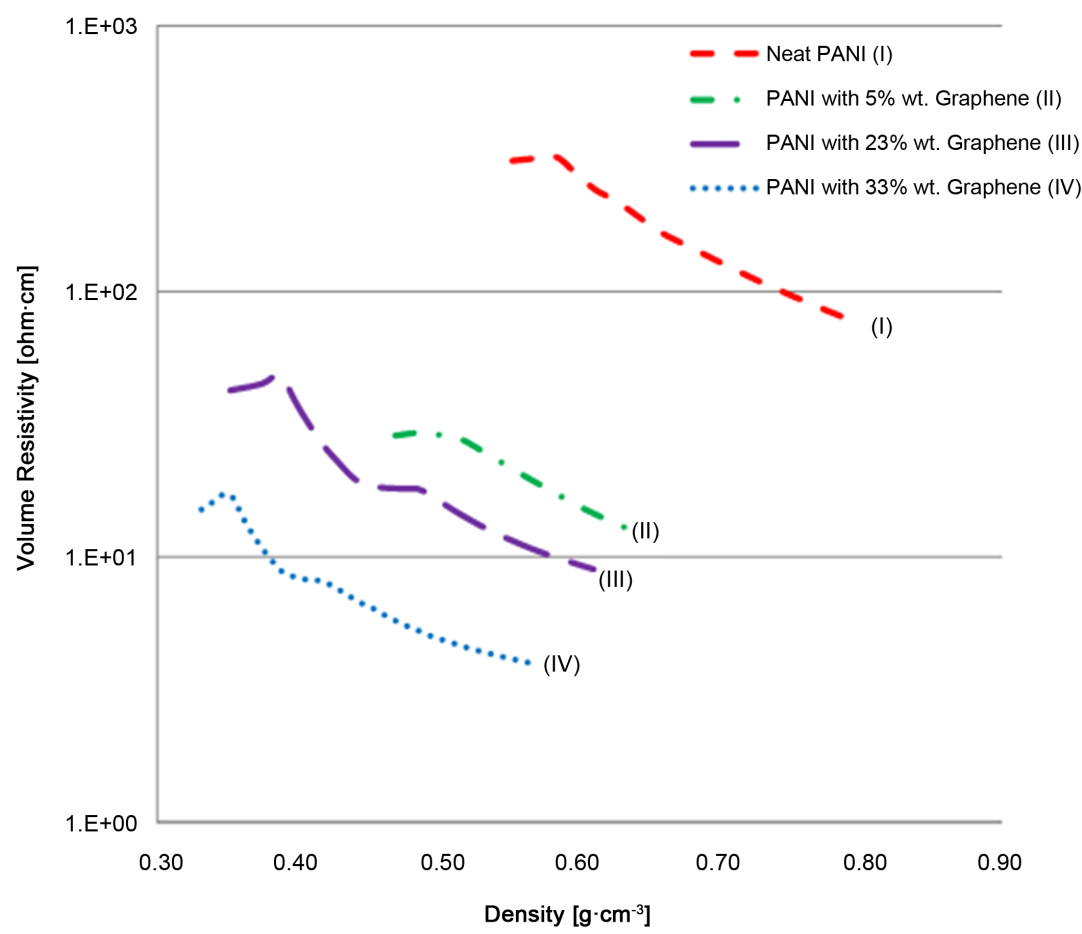

(b)

Figure 6. (a) PANI/graphene powders resistivity dependence on the compression pressure; (b) PANI/graphene powders resistivity dependence on density. 
Figure 6(b) demonstrates the nanocomposites powders volume resistivity dependence on density. A decrease in resistivity is demonstrated with the increase of the graphene content, similar to Figure 3 . This trend is kept for the various concentrations, as density increases the resistivity decreases due to particle rearrangement and better contacts [47]. Also, since graphene sheets are significantly more rigid than the neat PANI matrix [48] [49], they are less densified than neat PANI at the same applied pressure $(1600 \mathrm{kPa})$. The final density of neat graphene and neat PANI is $\sim 0.55 \mathrm{~g} \cdot \mathrm{cm}^{-3}$ and $\sim 0.79 \mathrm{~g} \cdot \mathrm{cm}^{-3}$, respectively.

Figure 7 depicts resistivity dependence on graphene content for the powder compacts comprssed under a load of $6 \mathrm{MPa}$. Resistivity values significantly decrease, by 2 orders of magnitude, from $\sim 550 \Omega \cdot \mathrm{cm}$ for the neat PANI down to $\sim 8 \Omega \cdot \mathrm{cm}$ for PANI with $41 \%$ wt. graphene. As previously suggested, an increase in graphene concentration results in better contacts and pathways for current flow. At a graphene content of $\sim 10 \%$ wt. the trend of the graph is reaching a plateau. It can be concluded that this is approximately the maximum graphene concentration needed to produce contact points between graphene sheets embedded in the PANI matrix.

Figure 8 depicts the applied stress (Figure 8(a)), mechanical strain (Figure 8(b)) and the measured electrical resistivity (Figure 8(c)) in the cyclic measurments. In these measurements of the PANI/graphene powder nanocomposites, the following were studied: I) Neat PANI, II) PANI with 5\% wt. graphene, III) PANI with 23\% wt. graphene, IV) PANI with 33\% wt. graphene and V) Neat graphene. A strong dependence of the applied stress, the measured strain and the electrical resistivity is shown. There is a direct, consistent and reproducible connection between the mechanical properties and the electrical resistance.

Figure 8(a) depicts the stress dependence on time. Stress is applied on the powder until a value of $\sim 1600 \mathrm{kPa}$ is reached, subsequently the stress is slowly removed until it is completely relieved and the cycle is then completed.

Figure 8(b) depicts the strain dependence on time. When stress is at a maximum value the strain is also in a maximum value and vise versa, as expected. It can be seen that the amplitude values of strain, after the particles are rearranged and a compacted sample is formed, remains approximately $2 \%-3 \%$ for all concentrations. It is suggested that this mechanical similarity behavior is determined by geometric parameters such as particle size and thus, are not intrinsic material properties. The strain values for the neat graphene are significantly higher than for the neat PANI and the other nanocomposite concentrations. The initial void fracture for graphene is much higher than in the other samples as shown in Figure 3(d), resulting in a higher strain and higher degree of compaction.

Figure 8(c) depicts the electrical resistivity dependence on time. As previously seen in Figure 6, the resistivity significantly decreases by the addition of graphene sheets. A decrease of two orders of magnitude for neat PANI and neat graphene is found. The addition of graphene lowers the resistivity, and further supports the suggestion that the graphene sheets create pathways for electric current conduction.

Figure 9 depicts a representative correlation of the experimental data for a $9 \%$ wt. graphene composite with

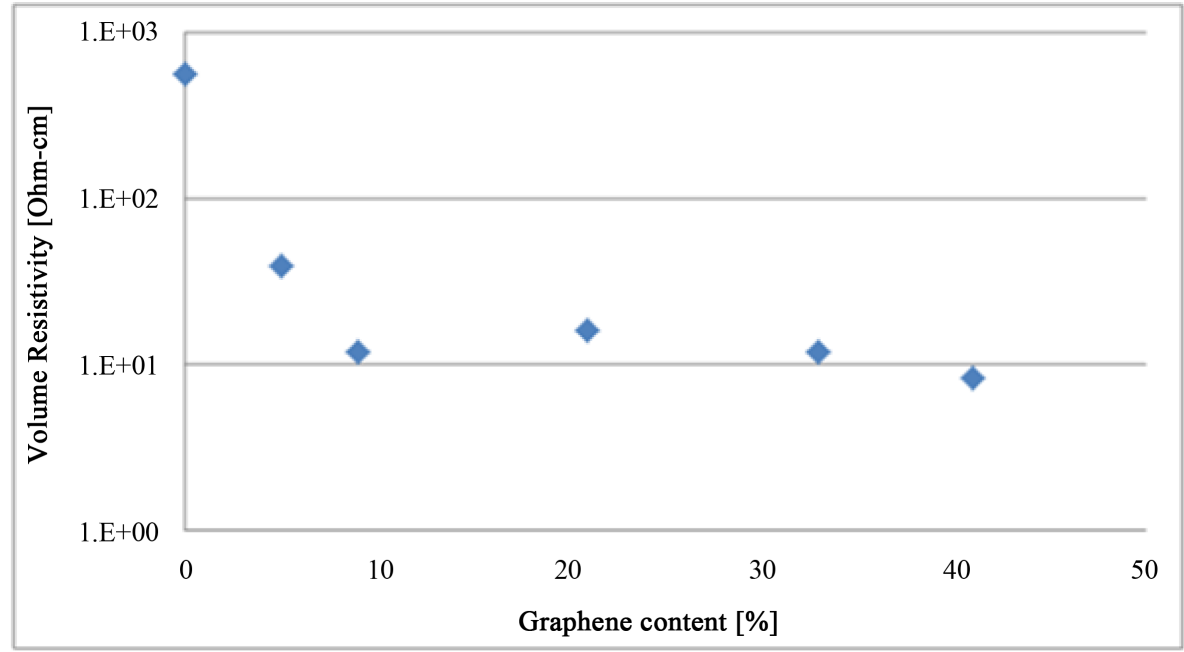

Figure 7. PANI/graphene powders compacts (compressed at a load of $6 \mathrm{MPa}$ ) bulk resistivity dependence on graphene content. 


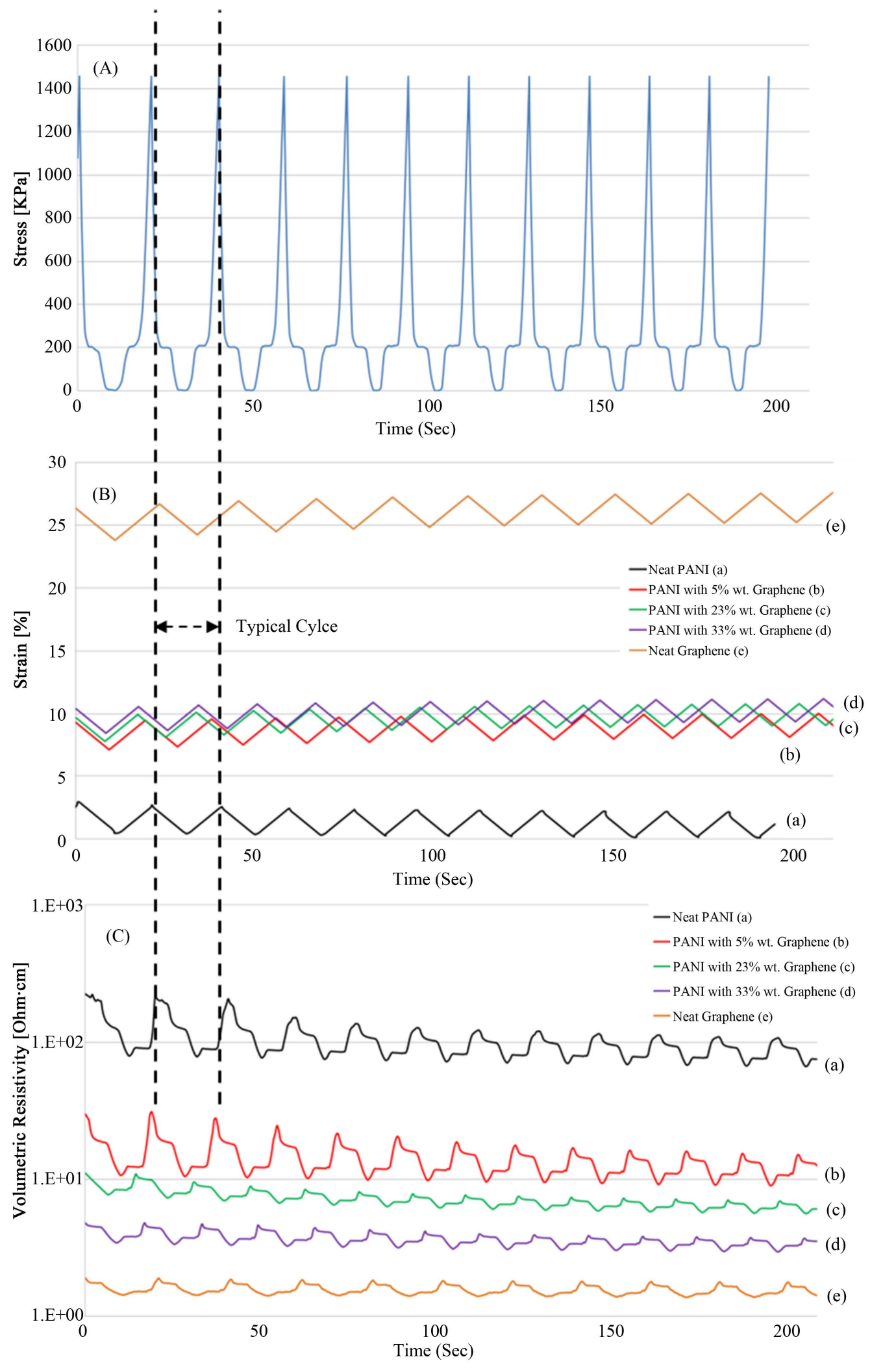

Figure 8. PANI/graphene powders cyclic measurements: (A) Stress dependence on time; (B) Strain dependence on time; (C) Electrical resistivity dependence on time, for: (a) Neat PANI; (b) PANI with 5\% wt. graphene; (c) PANI with 23\% wt. graphene; (d) PANI with 33\% wt. graphene; (e) Neat graphene. 


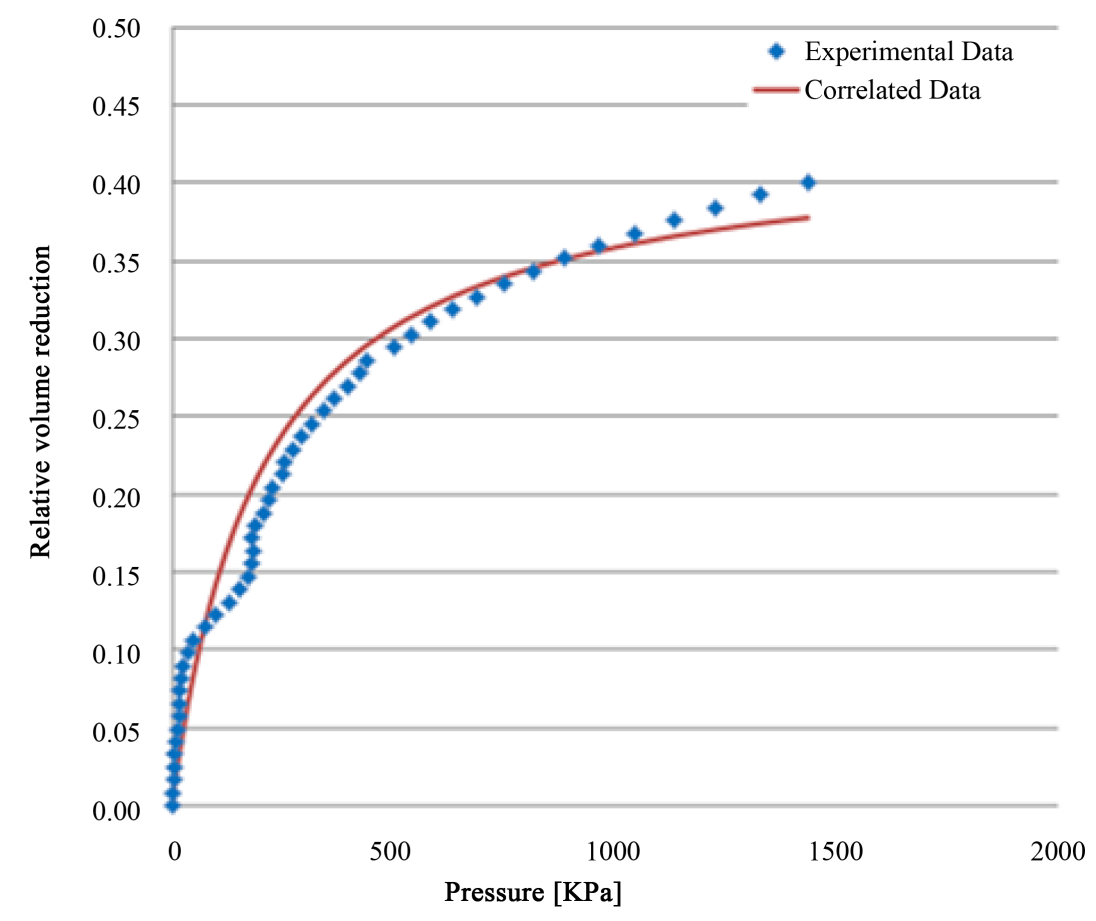

Figure 9. Volume reduction of PANI with 9\% wt. graphene, comparison of experimental data with the Kawakita model.

the Kawakita model [35]. The experimental results are well correlated to the model i.e. the model could be used to estimate the pressure needed to achieve a certain volume reduction for the PANI/graphene nanocomposites. This prediction along with the results of Figure 4 could link the applied pressure on the powder nanocomposites and the electrical resistivity of these nanocomposites via the change in volume or density.

In summary, this paper presents a novel sensing material prepared from PANI/graphene nanocomposites. The composites are prepared using the inverse emulsion polymerization method resulting in both a good dispersion of the graphene sheets in the PANI matrix and encapsulating the graphene sheets with PANI. The electrical resistivity is significantly lowered, by two orders of magnitude, for both the powder and the compacted powder samples by addition of graphene sheets. Cyclic measurements of applying/removing the stress on the nanocomposite specimens have shown a direct and clear dependence of strain and electrical resistivity. The PANI/graphene nanocomposites' volume reduction is well correlated to the Kawakita model which predicts volume reduction as function of applied pressure in compression experiments. Gauge factor values for the nanocomposites vary from $\sim 2.5$ to $\sim 2$ for the neat PANI and PANI with $41 \%$ wt. graphene, respectively.

\section{Conclusion}

This paper describes an in-situ inverse emulsion polymerization method of aniline in the presence of graphene sheets under sonication, resulting in PANI/graphene nanocomposite dispersions. The graphene sheets are well dispersed in the PANI matrix and are actually coated with PANI. One can conclude from the electrical measurements that the graphene sheets significantly lower, by two orders of magnitude, the electrical resistivity of the composites of both the powder and the compacted samples. With the increase of graphene content, the resistivity decreases. Cyclic experiments show that when pressure is applied the resistivity decreases, and when removed the resistivity increases and approximately resumes the former value. This result repeats itself consistently for all graphene contents demonstrating a principle that can be used as a sensing component for electromechanical sensors.

\section{Acknowledgements}

The authors are grateful for the financial assistance provided by the Russell Berrie Nanotechnology Institute 
(RBNI), Technion-IIT.

The authors also wish to thank Carmel olefins Ltd. and Dr. Genadi Shter of the Chemical Engineering Dept., Technion-ITT for their assistance.

\section{References}

[1] Mukhopadhyay, P. and Gupta, R.K. (2012) Graphite, Graphene, and Their Polymer Nanocomposites. CRC, Boca Raton. http://dx.doi.org/10.1201/b13051

[2] Rosen-Kligvasser, J., Suckeveriene, R.Y., Tchoudakov, R. and Narkis, M. (2013) A Novel Methodology for Controlled Migration of Antifog from Thin Polyolefin Films. Polymer Engineering \& Science, 54, 2023-2028. http://dx.doi.org/10.1002/pen.23755

[3] Peng, R., Wang, Y., Tang, W., Yang, Y. and Xie, X. (2013) Progress in Imidazolium Ionic Liquids Assisted Fabrication of Carbon Nanotube and Graphene Polymer Composites. Polymers, 5, 847-872.

http://dx.doi.org/10.3390/polym5020847

[4] Fakirov, S. (2013) Nano- and Microfibrillar Single-Polymer Composites: A Review. Macromolecular Materials and Engineering, 298, 9-32. http://dx.doi.org/10.1002/mame.201200226

[5] Byrne, M.T. and Gun'ko, Y.K. (2010) Recent Advances in Research on Carbon Nanotube-Polymer Composites. Advanced Materials, 22, 1672-1688. http://dx.doi.org/10.1002/adma.200901545

[6] Paul, D.R. and Robeson, L.M. (2008) Polymer Nanotechnology: Nanocomposites. Polymer, 49, 3187-3204. http://dx.doi.org/10.1016/j.polymer.2008.04.017

[7] Suckeveriene, R.Y., Mechrez, G., Filiba, H.O., Mosheev, S. and Narkis, M. (2012) Synthesis of Hybrid Polyaniline/ Carbon Nanotubes Nanocomposites in Toluene by Dynamic Interfacial Inverse Emulsion Polymerization under Sonication. Journal of Applied Polymer Science, 128, 2129-2135.

[8] Geim, A.K. and Novoselov, K.S. (2007) The Rise of Graphene. Nature Materials, 6, 183-191. http://dx.doi.org/10.1038/nmat1849

[9] Novoselov, K.S., Geim, A.K, Morozov, S.V., Jiang, D., Zhang, Y., Dubonos, S.V., Grigorieva, I.V. and Firsov, A.A. (2004) Electric Field Effect in Atomically Thin Carbon Films. Science, 306, 666-669. http://dx.doi.org/10.1126/science.1102896

[10] Novoselov, K.S., Geim, A.K, Morozov, S.V., Jiang, D., Katsnelson, M.I., Grigorieva, I.V., Dubonos, S.V. and Firsov, A.A. (2005) Two-Dimensional Gas of Massless Dirac Fermions in Graphene. Nature, 438, 197-200. http://dx.doi.org/10.1038/nature04233

[11] Kuila, T., Bose, S., Mishra, A.K., Khanra, P., Kim, N.H. and Lee, J.H. (2012) Chemical Functionalization of Graphene and Its Applications. Progress in Materials Science, 57, 1061-1105. http://dx.doi.org/10.1016/j.pmatsci.2012.03.002

[12] Ebrahimi, F. (2012) Nanocomposites—New Trends and Developments. InTech, Rijeka. http://dx.doi.org/10.5772/3389

[13] Nicolais, L., Borzacchiello, A. and Lee, S.M. (2012) Wiley Encyclopedia of Composites. 2nd Edition, John Wiley \& Sons, Inc., Hoboken.

[14] Ciric-Marjanovic, G. (2013) Recent Advances in Polyaniline Research: Polymerization Mechanisms, Structural Aspects, Properties and Applications. Synthetic Metals, 177, 1-47. http://dx.doi.org/10.1016/j.synthmet.2013.06.004

[15] Sapurina, I. and Stejskal, J. (2008) The Mechanism of the Oxidative Polymerization of Aniline and the Formation of Supramolecular Polyaniline Structures. Polymer International, 57, 1295-1325. http://dx.doi.org/10.1002/pi.2476

[16] Leea, H.Y., Rwei, S.P., Wang, L. and Chen, P.H. (2008) Preparation and Characterization of Core-Shell PolyanilinePolystyrene Sulfonate@ $\mathrm{Fe}_{3} \mathrm{O}_{4}$ Nanoparticles. Materials Chemistry and Physics, 112, 805-809. http://dx.doi.org/10.1016/j.matchemphys.2008.06.050

[17] Li, Z.H. and Wang, Y.W. (2010) Characterization of Polyaniline/Ag Nanocomposites Using $\mathrm{H}_{2} \mathrm{O}_{2}$ and Ultrasound Radiation for Enhancing Rate. Polymer Composites, 31, 1662-1668. http://dx.doi.org/10.1002/pc.20956

[18] Jeon, I.Y., Tan, L.S. and Baek, J.B. (2010) Synthesis and Electrical Properties of Polyaniline/Polyaniline Grafted Multiwalled Carbon Nanotube Mixture via in Situ Static Interfacial Polymerization. Journal of Polymer Science Part A: Polymer Chemistry, 48, 1962-1972. http://dx.doi.org/10.1002/pola.23963

[19] Li, S., Gan, M., Ma, L., Yan, J., Tang, J., Fu, D., Li, Z. and Bai, Y. (2013) Preparation and Microwave Absorbing Properties of Polyaniline-Modified Silicon Carbide Composites. High Performance Polymers, 25, 901-906. http://dx.doi.org/10.1177/0954008313487393

[20] Suckeveriene, R.Y., Zelikman, E., Mechrez, G., Tzur, A., Frisman, I., Cohen, Y. and Narkis, M. (2011) Synthesis of Hybrid Polyaniline/Carbon Nanotube Nanocomposites by Dynamic Interfacial Inverse Emulsion Polymerization under Sonication. Journal of Applied Polymer Science, 120, 676-682. http://dx.doi.org/10.1002/app.33212 
[21] Wang, H., Hao, Q., Yang, X., Lu, L. and Wang, X. (2010) A Nanostructured Graphene/Polyaniline Hybrid Material for Supercapacitors. Nanoscale, 2, 2164-2170. http://dx.doi.org/10.1039/c0nr00224k

[22] Fan, Y., Liu, J.H., Yang, C.P., Yu, M. and Liu, P. (2011) Graphene-Polyaniline Composite Film Modified Electrode for Voltammetric Determination of 4-Aminophenol. Sensors and Actuators B, 157, 669-674. http://dx.doi.org/10.1016/j.snb.2011.05.053

[23] Radhapyari, K., Kotoky, P., Das, M.R. and Khan, R. (2013) Graphene-Polyaniline Nanocomposite Based Biosensor for Detection of Antimalarial Drug Artesunate in Pharmaceutical Formulation and Biological Fluids. Talanta, 111, 47-53. http://dx.doi.org/10.1016/j.talanta.2013.03.020

[24] Salavagione, H.J., Martinez, G. and Ellis, G. (2011) Recent Advances in the Covalent Modification of Graphene with Polymer. Macromolecular Rapid Communications, 32, 1771-1789. http://dx.doi.org/10.1002/marc.201100527

[25] Bai, H., Xu, Y., Zhao, L., Li, C. and Shi, G. (2009) Non-Covalent Functionalization of Graphene Sheets by Sulfonated Polyaniline. Chemical Communications, 13, 1667-1669. http://dx.doi.org/10.1039/b821805f

[26] Tkalya, E.E., Ghislandi, M., de With, G. and Koning, C.E. (2012) The Use of Surfactants for Dispersing Carbon Nanotubes and Graphene to Make Conductive Nanocomposites. Current Opinion in Colloid \& Interface Science, 17, 225232. http://dx.doi.org/10.1016/j.cocis.2012.03.001

[27] Shan, C., Yang, H., Han, D., Zhang, Q., Ivaska, A. and Niu, L. (2009) Water-Soluble Graphene Covalently Functionalized by Biocompatible Poly-L-Lysine. Langmuir, 25, 12030-12033. http://dx.doi.org/10.1021/la903265p

[28] Odian, G. (2004) Principles of Polymerization. 4th Edition, John Wiley \& Sons, Inc., Hoboken. http://dx.doi.org/10.1002/047147875X

[29] Bovey, F.A., Kolthoff, I.M., Medalia, A.I. and Meehan, E.J. (1955) Emulsion Polymerization. Interscience Publishers, Inc., New York.

[30] Zelikman, E., Suckeveriene, R.Y., Mechrez, G. and Narkis, M. (2010) Fabrication of Composite Polyaniline/CNT Nanofibers Using an Ultrasonically Assisted Dynamic Inverse Emulsion Polymerization Technique. Polymers for Advanced Technologies, 21, 150-152.

[31] Nordstrom, J., Klevan, I. and Alderborn, G. (2012) A Protocol for the Classification of Powder Compression Characteristics. European Journal of Pharmaceutics and Biopharmaceutics, 80, 209-216. http://dx.doi.org/10.1016/j.ejpb.2011.09.006

[32] Montes, J.M., Cuevas, F.G., Cintas, J. and Urban, P. (2011) Electrical Conductivity of Metal Powders under Pressure. Applied Physics A, 105, 935-947. http://dx.doi.org/10.1007/s00339-011-6515-9

[33] Han, B.G., Han, B.Z. and Ou, J.P. (2009) Experimental Study on Use of Nickel Powder-Filled Portland Cement-Based Composite for Fabrication of Piezoresistive Sensors with High Sensitivity. Sensors and Actuators A: Physical, 149, 51-55. http://dx.doi.org/10.1016/j.sna.2008.10.001

[34] Han, B.G., Yu, Y., Han, B.Z. and Ou, J.P. (2008) Development of a Wireless Stress/Strain Measurement System Integrated with Pressure-Sensitive Nickel Powder-Filled Cement-Based Sensors. Sensors and Actuators A: Physical, 147, 536-543. http://dx.doi.org/10.1016/j.sna.2008.06.021

[35] Kawakita, K. and Tsutsmui, Y. (1966) A Comparison of Equations for Powder Compression. Bulletin of the Chemical Society of Japan, 39, 1364-1368. http://dx.doi.org/10.1246/bcsj.39.1364

[36] Hauptmann, P. (1993) Sensors Principles and Applications. Carl Hanser Verlag, Munich.

[37] Costa, P., Ferreira, A., Sencadas, V., Viana, J.C. and Lanceros-Mendez, S. (2013) Electro-Mechanical Properties of Triblock Copolymer Styrene-Butadiene-Styrene/Carbon Nanotube Composites for Large Deformation Sensor Applications. Sensors and Actuators A, 201, 458-467. http://dx.doi.org/10.1016/j.sna.2013.08.007

[38] Hwang, S.H., Park, H.W. and Park, Y.B. (2013) Piezoresistive Behavior and Multi-Directional Strain Sensing Ability of Carbon Nanotube-Graphene Nanoplatelet Hybrid Sheets. Smart Materials and Structures, 22, Article ID: 015013. http://dx.doi.org/10.1088/0964-1726/22/1/015013

[39] Li, W., He, D. and Bai, J. (2014) The Influence of Nano/Micro Hybrid Structure on the Mechanical and Self-Sensing Properties of Carbon Nanotube-Microparticle Reinforced Epoxy Matrix Composite. Composites Part A, 54, 28-36. http://dx.doi.org/10.1016/j.compositesa.2013.07.002

[40] Ku-Herrera, J.J., Aviles, F. and Seidel, G.D. (2013) Self-Sensing of Elastic Strain, Matrix Yielding and Plasticity in Multiwall Carbon Nanotube/Vinyl Ester Composites. Smart Materials and Structures, 22, Article ID: 085003. http://dx.doi.org/10.1088/0964-1726/22/8/085003

[41] Talmon, Y. (1999) Cryogenic Temperature Transmission Electron Microscopy in the Study of Surfactant Systems. Surfactant Science Series, 83, 147-178.

[42] Kim, D.K., Oh, K.W. and Kim, S.H. (2008) Synthesis of Polyaniline/Multiwall Carbon Nanotube Composite via Inverse Emulsion Polymerization. Journal of Polymer Science Part B: Polymer Physics, 46, 2255-2266. 
http://dx.doi.org/10.1002/polb.21557

[43] Haba, Y., Segal, E., Narkis, M., Titelman, G.I. and Siegmann, A. (1999) Polymerization of Aniline in the Presence of DBSA in an Aqueous Dispersion. Synthetic Metals, 106, 59-66. http://dx.doi.org/10.1016/S0379-6779(99)00100-9

[44] Oyefusia, A., Olanipekuna, O., Neelgund, G.M., Peterson, D., Stone, J.M., Williams, E., Carson, L., Regisfor, G. and Oki, A. (2014) Hydroxyapatite Grafted Carbon Nanotubes and Graphene Nanosheets: Promising Bone Implant Materials. Spectrochimica Acta Part A: Molecular and Biomolecular Spectroscopy, 132, 410-416. http://dx.doi.org/10.1016/j.saa.2014.04.004

[45] Ran, S., Chen, C., Guo, Z. and Fang, Z. (2014) Char Barrier Effect of Graphene Nanoplatelets on the Flame Retardancy and Thermal Stability of High-Density Polyethylene Flame-Retarded by Brominated Polystyrene. Journal of Applied Polymer Science, 131, 40520. http://dx.doi.org/10.1002/app.40520

[46] Xu, J., Liu, J. and Li, K. (2014) Application of Functionalized Graphene Oxide in Flame Retardant Polypropylene. Journal of Vinyl and Additive Technology, Early View. http://dx.doi.org/10.1002/vnl.21415

[47] Marinho, B., Ghislandi, M., Tkalya, E., Koning, C.E. and de With, G. (2012) Electrical Conductivity of Compacts of Graphene, Multi-Wall Carbon Nanotubes, Carbon Black, and Graphite Powder. Powder Technology, 221, 351-358. http://dx.doi.org/10.1016/j.powtec.2012.01.024

[48] Wang, D.W., Li, F., Zhao, J., Ren, W., Chen, Z.G., Tan, J., Wu, Z.S., Gentle, I., Lu, G.Q. and Cheng, H.M. (2009) Fabrication of Graphene/Polyaniline Composite Paper via in Situ Anodic Electro Polymerization for High-Performance Flexible Electrode. ASC Nano, 3, 1745-1752.

[49] Valentova, H. and Stejskal, J. (2010) Mechanical Properties of Polyaniline. Synthetic Metals, 160, 832-834. http://dx.doi.org/10.1016/j.synthmet.2010.01.007 
Scientific Research Publishing (SCIRP) is one of the largest Open Access journal publishers. It is currently publishing more than 200 open access, online, peer-reviewed journals covering a wide range of academic disciplines. SCIRP serves the worldwide academic communities and contributes to the progress and application of science with its publication.

Other selected journals from SCIRP are listed as below. Submit your manuscript to us via either submit@scirp.org or Online Submission Portal.
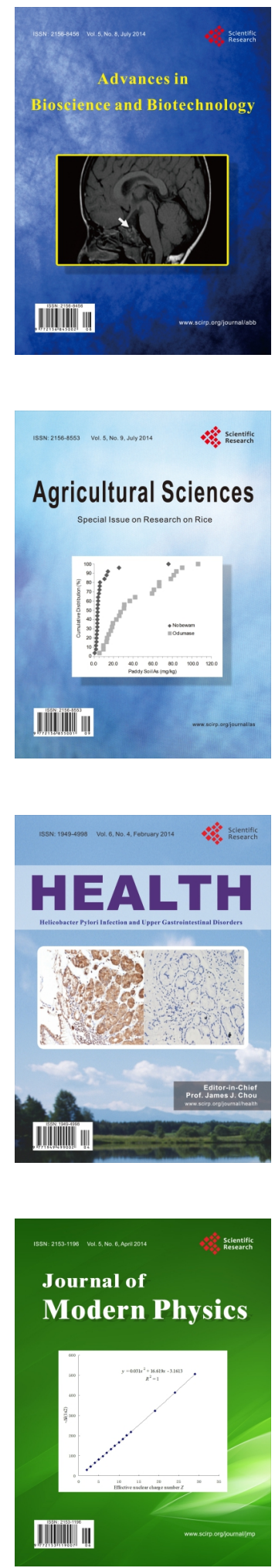
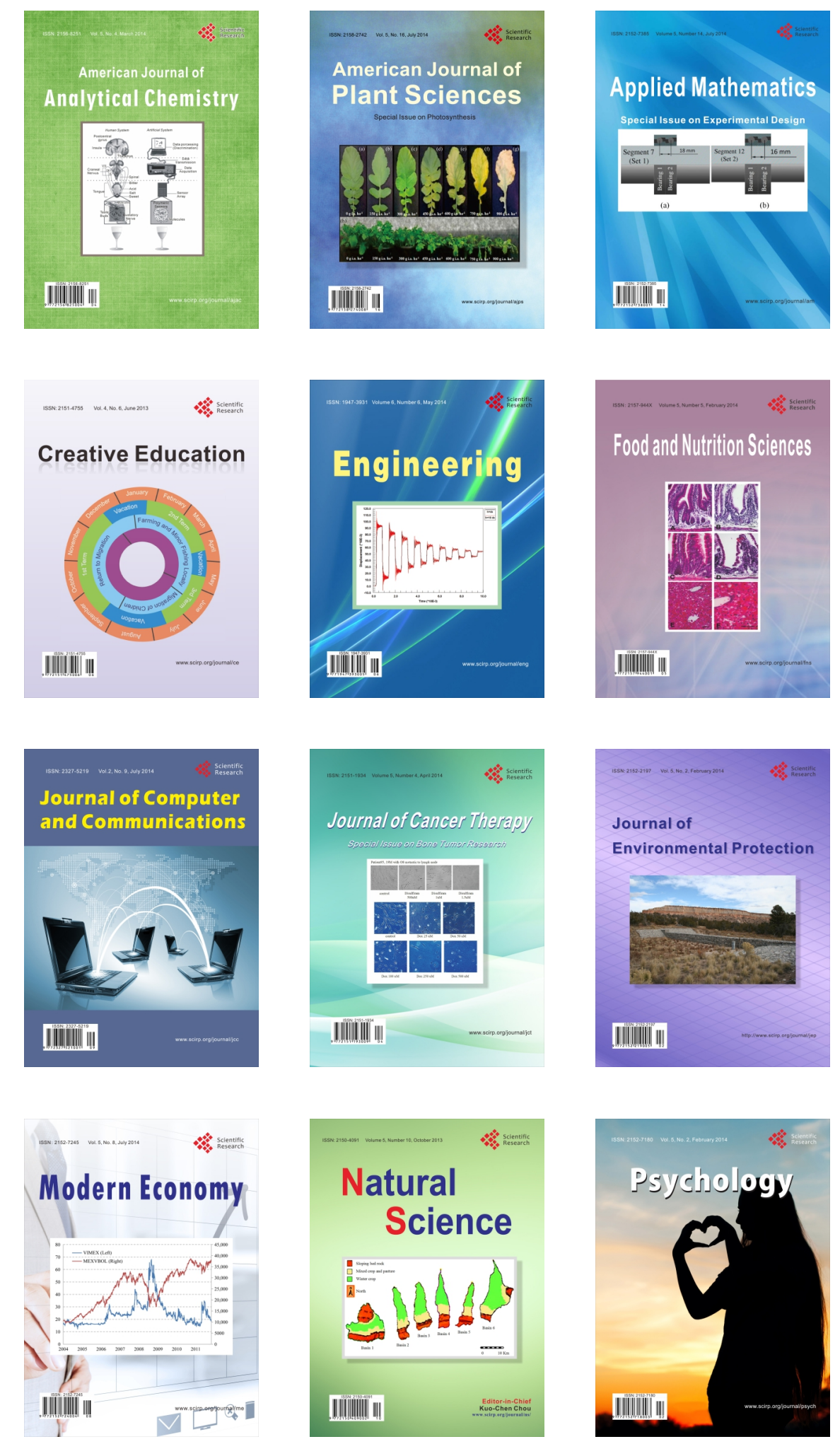